Int. J. Dev. Biol. 58: 713-717 (2014)

doi: $10.1387 / \mathrm{ijdb} .140094 \mathrm{jd}$

\title{
Regional divergence of palate medial edge epithelium along the anterior to posterior axis
}

\author{
JIU-ZHEN JIN, DENNIS R. WARNER and JIXIANG DING* \\ Department of Molecular, Cellular \& Craniofacial Biology, School of Dentistry and Birth Defects Center, \\ University of Louisville, Louisville, KY, USA
}

\begin{abstract}
Recent studies have shown that mouse palatal mesenchymal cells undergo regional specification along the anterior-posterior (A-P) axis defined by anterior Shox 2 and Msx 1 expression and posterior Meox2 expression. A-P regional specification of the medial edge epithelium, which is directly responsible for palate fusion, has long been proposed, but it has not yet been demonstrated due to the lack of regional specific markers. In this study, we have demonstrated that the palate medial edge epithelium is regionalized along the A-P axis, similar to that for the underlying mesenchyme. Mmp13, a medial edge epithelium specific marker, was uniformly expressed from anterior to posterior in wild-type mouse palatal shelves. Previous studies demonstrated that medial edge epithelium expression of $M m p 13$ was regulated by TGF- $\beta 3$. We have found that the changes in $M m p 13$ expression in TGF- $\beta 3$ knockouts varied along the A-P axis, and can be broken down into three distinct regions. These regions correlated with regional specification of the underlying medial edge mesenchymal cells and timing of palate fusion. Mouse palate medial edge epithelium along the A-P axis can be divided into different regions according to the differential response to the loss of TGF- $\beta 3$.
\end{abstract}

KEY WORDS: Mmp13, TGF- $\beta 3$, mouse secondary palate, regional specification

Mammalian palatogenesis is a complex developmental process in which the bilateral palate shelves fuse along the facial midline to form the continuous palate that separates the oral and nasal cavities (Bush and Jiang, 2012, Ferguson, 1988, Murray and Schutte, 2004, Nawshad et al., 2004). Each nascent palatal shelf is made up of a core of neural crest-derived mesenchymal cells enclosed by multiple layers of ectoderm-derived epithelial sheets (Bush and Jiang, 2012, Chai and Maxson, 2006, Ferguson, 1988). In mice, between embryonic day 12.5 and 13.5 (E12.5-E13.5) the two developing palatal shelves first grow in a vertical direction lateral to the tongue. On E14.5, however, the vertical palatal shelves re-orient to form horizontal shelves above the dorsal side of the tongue. The two horizontal palatal shelves continue to grow until they meet each other at their medial edge epithelium (MEE) areas (Bush and Jiang, 2012, Chai and Maxson, 2006, Ferguson, 1988). MEE contact induces a series of cellular events that culminates in the elimination of the epithelial seam formed from the union of the palatal shelves. This medial edge seam (MES) disappears by E15.5 leading to the mesenchymal confluence of the definitive palate (Carette and Ferguson, 1992, Griffith and Hay, 1992, Shuler et al., 1992). MEE cells are critical players in the process of fu- sion and their differentiation is determined in part by signals from the underlying medial edge mesenchymal cells as demonstrated by tissue recombination experiments (Ferguson et al., 1984). In parallel with vertical and horizontal growth, the palatal shelves also extend along the A-P axis as the head develops (Li and Ding, 2007, Welsh and O'Brien, 2009). Moreover, the palatal shelf exhibits A-P regional differentiation as well as A-P growth. The morphological, cellular and molecular differences between anterior and posterior palatal mesenchymal cells are quite evident and therefore are better understood than those of the MEE. Anterior palate mesenchymal cells will form the bony palate through ossification and most of the posterior mesenchymal cells will become smooth muscle cells and form the soft palate (Cui et al., 2005, Ferguson, 1988). Recently, A-P regional specification of palatal mesenchymal cells has been studied at the molecular level (Hilliard et al., 2005). Mouse homeobox gene $M s x 1$ is expressed only in anterior palatal mesenchymal cells (Hilliard et al., 2005, Zhang et al., 2002), and is required for palate growth because loss of Msx 1 function in mice

Abbreviations used in this paper: A-P, anterior-posterior; E14.5/15.5, embryonic day 14.5/15.5; MEE, medial edge epithelium; R, rugae; WT, wild type.

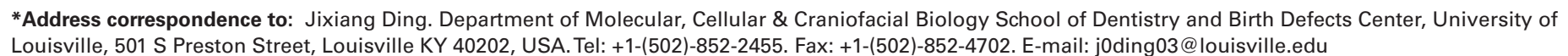

Accepted: 10 September 2014.

ISSN: Online 1696-3547, Print 0214-6282

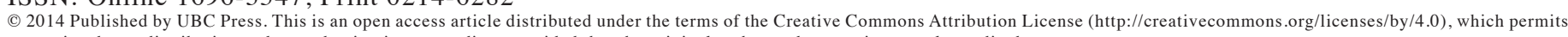
unrestricted use, distribution and reproduction in any medium provided that the original author and source is properly credited.

Printed in Spain. 

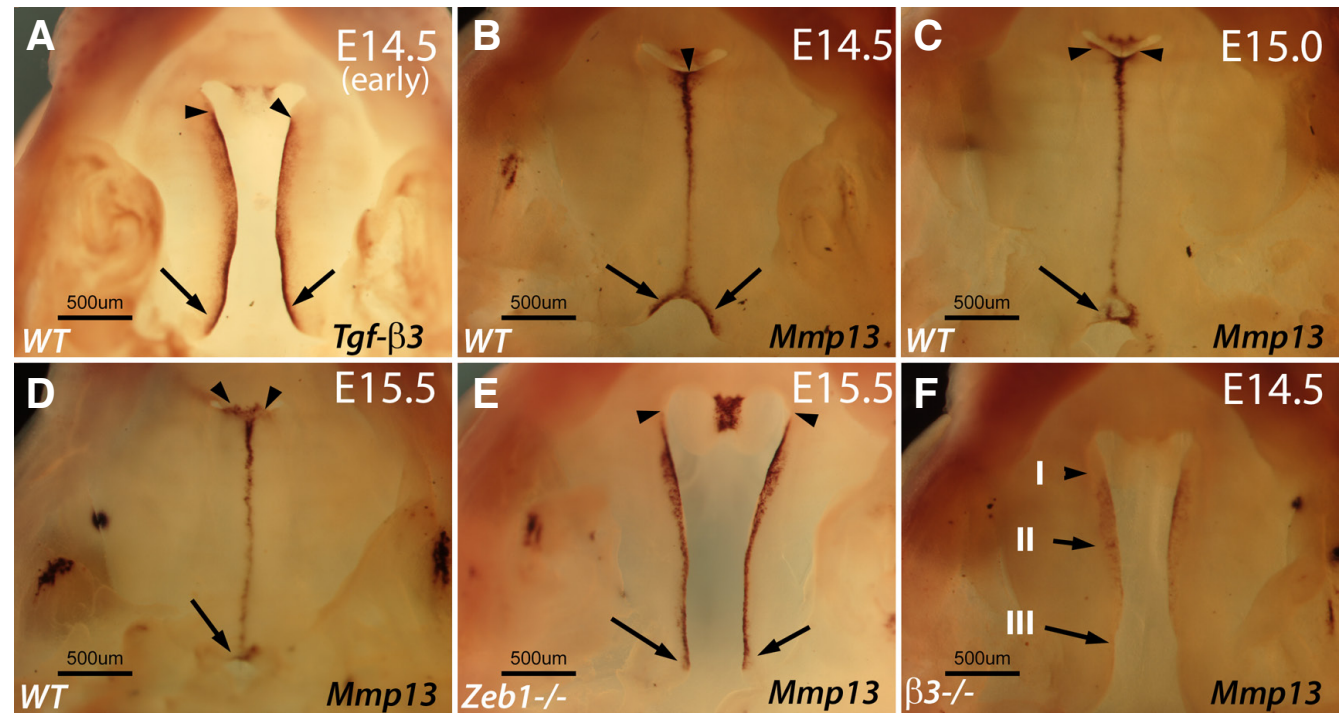

Fig. 1. Whole mount in situ hybridization showing the expression of Tgf- $\beta 3$ (A) and Mmp13 (B-F) in wild type (A-D), Zeb1 mutant (E) and Tgf- $\beta 3$ mutant (F) embryos on E14.5 (A, B and F), E15.0 (C) and E15.5 (D and E). Note that there was differential expression of Mmp13 in Tgf- $\beta 3$ mutant palatal shelves along the A-P axis as indicated in panel F (regions of I, II, and III). Scale bars, 500 um.

leads to cleft palate due to a growth defect (Satokata and Maas, 1994, Zhang et al., 2002). The cleft palate defect in Msx1 mutant embryos can be rescued by trans-expression of the Bmp4 gene, indicating BMP4 functions downstream of MSX1 in palate growth control (Zhang et al., 2002). Moreover, the proliferation of anterior, but not the posterior, palatal mesenchymal cells are responsive to BMP treatment (Zhang et al., 2002). Similar to Msx1, Shox2 is another homeobox gene expressed only in the anterior palate, and loss of its expression in mice leads to an anterior cleft palate (Yu et al., 2005).

Unlike Msx1 and Shox2, the expression of Meox2, another homeobox gene, displays expression specifically in the posterior palate and $23 \%$ of Meox 2 mutant embryos have posterior cleft palate due to a post-fusion defect (Jin and Ding, 2006, Li and Ding, 2007).

Furthermore, both Shox2 and Meox2 genes have dynamic expression patterns in palate development. Shox2 is expressed initially in a small anterior palate domain that gradually expands to include the anterior two-thirds at later stages (Li and Ding, 2007). In contrast, Meox2 is initially expressed along the entire palate but regresses to a pattern that includes only the posterior, soft palate ( $\mathrm{Li}$ and Ding, 2007). This expression shift might be caused by regional cell proliferation (Welsh and O'Brien, 2009), mesenchymal cell migration (He et al., 2008) or cell type switch (Li and Ding, 2007).

In marked contrast to palatal mesenchymal cells, little is known about the A-P regional specification of the MEE, the cells directly responsible for fusion of the palatal shelves. This has likely been hampered due to the lack of A-P regional specific MEE markers.

\section{Results and Discussion}

Previous studies have shown that Tgf- $\beta 3$ is highly expressed in palate epithelium and is required for MEE differentiation and fu-
A

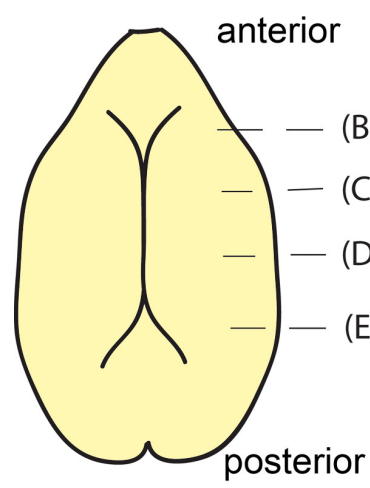

(B)

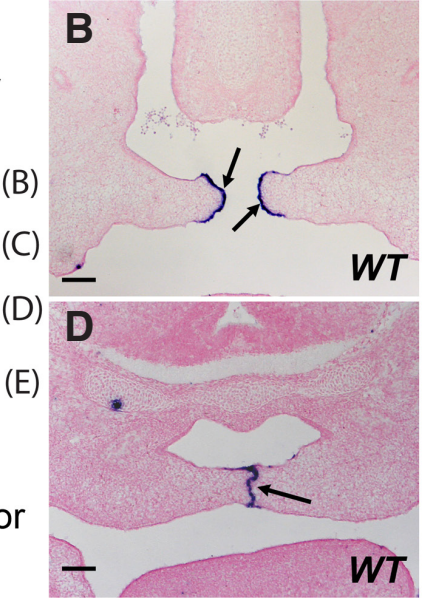

B
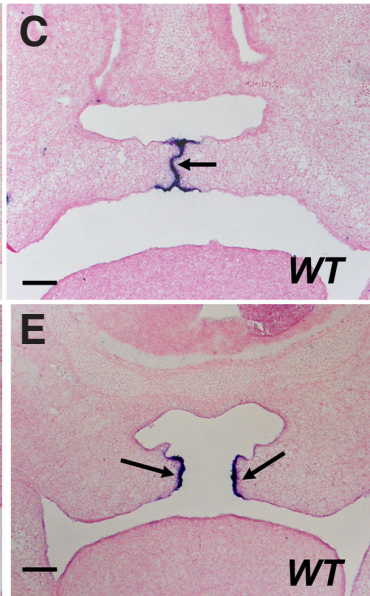

Fig. 2. Section in situ hybridization showing Mmp13is highly expressed in palate medial edge epithelium (MEE, arrows) from anterior to posterior in E14.5 wild type embryos. The dashed lines in (A) indicate the positions of panels (B,C,D,E). Scale bars, $100 \mu \mathrm{m}$.

sion (Fitzpatrick et al., 1990, Kaartinen et al., 1995, Pelton et al., 1990, Proetzel et al., 1995). Mmp13 is expressed during mouse palate development, exclusively in MEE cells on E14.5 and E15.5 (Blavier et al., 2001, Jin et al., 2008, Jin et al., 2010). This expression is regulated by TGF- $\beta 3$ because Mmp13 expression in Tgf- $\beta 3$ mutant MEE cells is significantly decreased (Blavier et al., 2001). To investigate the A-P regional specification of the MEE during mouse palate development, we first examined the expression of Tgf- $\beta 3$ and Mmp13 in mouse palate shelves by whole-mount in situ hybridization and found that both mRNAs were uniformly expressed in the MEE from anterior to posterior on E14.5 and E15.5. As shown in figure 1, on E14.5, the Tgf- $\beta 3$ expression domain covers the entire A-P axis of the palatal shelf (Fig. 1A) from the anterior (arrowheads in Fig. 1A) the posterior aspect (arrows in Fig. 1A). Correspondingly, the expression domain of Mmp13 also covers the 


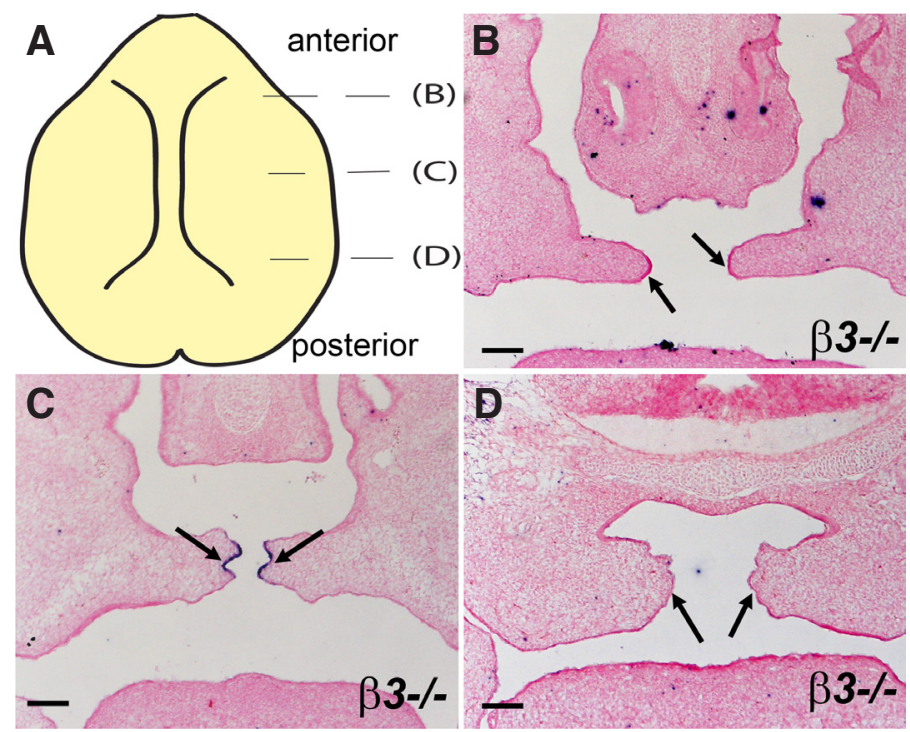

Fig. 3. Section in situ hybridization showing Mmp13 is expressed in the palate medial edge epithelium (MEE, arrows) only in the middle region (C), but not in the anterior $(\mathbf{B})$ and posterior (D) regions in E14.5 Tgf- $\beta 3$ mutant embryos. The dashed lines in (A) indicate the positions of panels $(B, C, D)$. Scale bars, $100 \mu \mathrm{m}$.

entire A-P axis from anterior end (arrowhead in Fig. 1B) to the soft palate (arrows in Fig. 1B). On E15.0, the expression of Mmp13 further extends into the anterior margin (arrowheads in Fig. 1C), and later in the primary palate on E15.5 (arrowheads in Fig. 1D). Section in situ hybridization also confirmed that the expression of Mmp13 in the MEE of wild type embryo was equally strong from the anterior to posterior aspect (Fig. 2). In Zeb1 mutant embryos, the two palatal shelves do not contact because of a delay in reorientation (Jin et al., 2008, Jin et al., 2010). However, Mmp13 was still highly expressed in the MEE from anterior end to posterior soft palate indicating that its expression was independent of palatal shelf contact (Fig. 1E) (Jin et al., 2008, Jin et al., 2010). As mentioned above previous studies demonstrated that Mmp13 expression is decreased in Tgf- $\beta 3$ mutant palatal shelves (Blavier et al., 2001). We therefore determined the expression of Mmp13 in Tgf- $\beta 3$ mutant embryos by whole-mount in situ hybridization. We observed that the change of $M m p 13$ expression in response to the loss of TGF- $\beta 3$ was dependent upon the specific location along the A-P axis (Fig. 1F). We have divided the MEE into three regions, based on Mmp13 expression in E14.5 Tgf- $\beta 3$ mutants (Fig. 1F): region I was negative for $M m p 13$ expression; region II shows weak expression of Mmp13; region III, corresponding to the soft palate, was negative for Mmp13 expression. Section in situ hybridization also confirmed that $M m p 13$ expression is present only in the middle region (Fig. $3 \mathrm{C}$ ), but absent in the anterior and posterior regions in Tgf- $\beta 3$ mutant palate shelves (Fig. $3 \mathrm{~B}$ and $D$, respectively).

To determine these regions more precisely, we determined Mmp13 expression in Tgf- $\beta 3$ mutant palatal shelves in relation to the position of the rugae, as determined by Shh expression (Fig. 4). E14.5 Tgf- $\beta 3$ mutant embryonic heads were bisected and one palatal shelf subjected to whole-mount in situ hybridization for Mmp13 expression and the other for Shh expression. The rugae were numbered based on their positions along the A-P axis according to the published report, in which the most anterior one is designated as ruga 1 (Economou et al., 2012). By comparing Mmp13expression with Shhexpression, we determined that region I, the anterior Mmp13 negative area, was located between rugae 1 and 2, region II, the middle Mmp13 positive area, was located between rugae 2 and 8 , and region III, the posterior Mmp13 negative area, corresponded to the entire soft palate (Fig. 5A and Fig. $6)$. On E15.5, region I of Tgf- $\beta 3$ mutant palatal shelves expressed low level Mmp13 similar to region II, whereas region III was devoid of Mmp13 expression (Fig. 5B).

Region III has the highest sensitivity to the loss of TGF- $\beta 3$, since it is negative for Mmp13 expression in Tgf- $\beta 3$ mutants on both E14.5 and E15.5 (Fig. 5 A and B). Region II, however, is the least sensitive to the loss of TGF- $\beta 3$, because it retains weak Mmp13 expression even in the absence of TGF- $\beta 3$ (Fig. 5 A,B).

Therefore, the MEE cells are indeed divergent along the A-P axis. Since tissue recombination experiments revealed that the MEE cells received signals from the underlying mesenchymal cells, we hypothesized that these mesenchymal cells were also divergent.
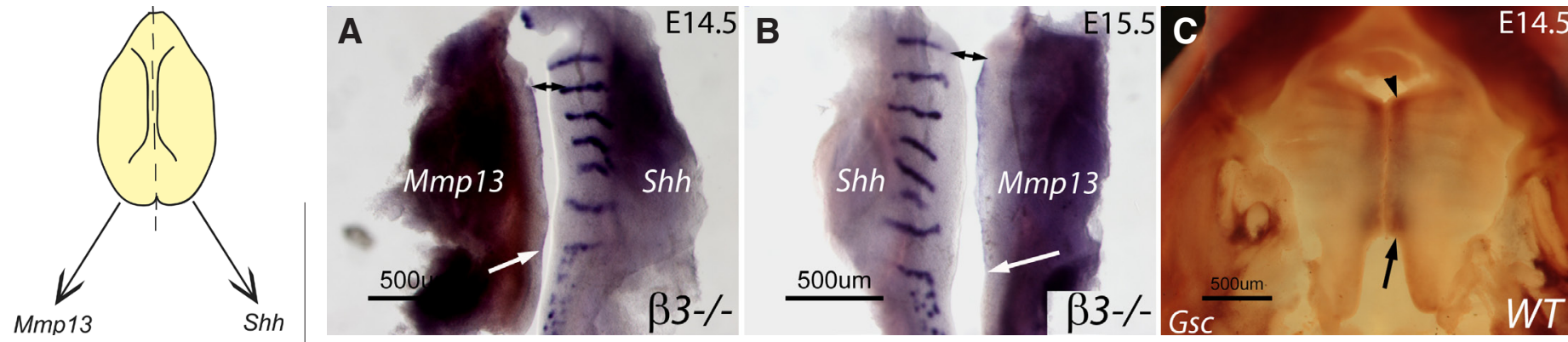

Fig. 4 (Left). Diagram showing the method to determine the Mmp13 expression domain in Tgf- $\beta 3$ mutant palatal shelves in relation to rugae as marked by Shh expression.

Fig. 5 (Right). Regional specification of medial edge epithelium in relation to the rugaes and subjacent mesenchymal cells. The anterior end of the Mmp13 expression domain in the Tgf- $\beta 3$ mutant palatal shelf at $E 14.5$ (A) resides approximately at ruga 2, see bi-arrowheads in (A) and the posterior end corresponded to ruga 8, see arrow in (A). At E15.5 (B), the anterior end of the Mmp13 expression domain extended to ruga 1, see bi-arrowheads in (B) and the posterior end stayed at the position of ruga 8. Rugae 1-8 is coincident with the anterior end, see arrowhead in (C) and posterior end of the Gsc expression domain (C). Scale bars represent $500 \mu \mathrm{m}$. 


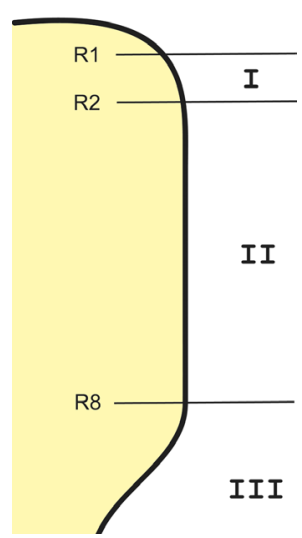

Fig. 6. Schematic representation of the three regions of the medial edge epithelium along the anterior-posterior axis identified by Mmp13 expression in Tgf- $\beta 3$ mutant embryos. R: rugae.

We determined the expression Goosecoid (Gsc), a marker for the cells underlying the medial edge (Jin et al., 2010, Proetzel et al., 1995) and found that it was present roughly in regions I and II, but not in region III based on the characteristic morphology of posterior soft palate (Fig. $5 \mathrm{C}$ ), suggesting that the medial edge mesenchymal cells adjacent to the MEE along the A-P axis were indeed different than cells located deeper in the mesenchyme and that this could determine MEE regional specification along $A-P$ axis.

It is interesting that MEE regional specification along the A-P axis correlates with the timing of palatal shelf fusion. Initial fusion of the palatal shelves begins within region II. Fusion then proceeds from this point both anteriorly and posteriorly (regions I and III, respectively). In addition, Tgf- $\beta 3$ mutant mice in certain background such as CF-1 strain display posterior cleft palate (Proetzel et al., 1995), the area corresponding to the region III, which is the most sensitive region to TGF- $\beta 3$ loss.

In summary, these data demonstrate that not only is there genespecific expression along the A-P axis for mesenchymal cells, but also for the medial edge epithelial cells suggesting a dynamic interaction between these two cell types that will likely play a role in fusion of the palatal shelves, perturbations of which will lead to clefts of the secondary palate.

\section{Materials and Methods}

\section{Mice}

We obtained the Tgf- $\beta 3$ mutant line on C57BL/6J background from the Jackson Laboratory, Maine, USA (stock number 002619), that was originally generated by Dr. Thomas Doetschman (Proetzel et al., 1995). Tgf- $\beta 3 \%$ mice have a complete anterior to posterior cleft palate (Proetzel et al., 1995). The Zeb1 mutant line has been previously reported by Dr. Yujiro Higashi (Takagi et al., 1998). Further analysis of the palate defects in this mutant were also described by Jin et al., (Jin et al., 2008, Jin et al., 2010).

\section{Examination of gene expression}

Whole mount in situ hybridization was carried out according to Shen (Shen, 2001). Briefly, C57BL/6 wild type mouse embryos and Tgf- $\beta 3$ and Zeb1 mutant embryos were dissected in cold PBS at the desired embryonic stages (the day when vaginal plugs were observed was designated as E0.5.). Heads were separated and the lower jaws removed and the remaining tissue was fixed in $4 \%$ paraformaldehyde in PBS overnight at $4^{\circ} \mathrm{C}$ followed by dehydration through $25 \%, 50 \%$ and $75 \%$ methanol in PBS containing $0.1 \%$ Tween 20 . The samples were stored in $100 \%$ methanol at $-20^{\circ} \mathrm{C}$ until used for in situ hybridization. The treated embryonic tissues were processed for non-radioactive whole mount in situ hybridization using digoxygenin-labeled antisense riboprobes as described by Shen (Shen, 2001). The hybridized embryos underwent post-hybridization fixation and were maintained in $80 \%$ glycerol for photography.

For the experiment described in figures 4 and 5 , more than 5 embryos at each stage were tested, and each embryo was marked by unique cuttings after bisection in order to be paired correspondingly after in situ hybridization.

Digoxygenin based section in situ hybridizations were carried out on cryo-sections according to Shen (Shen, 2001), followed by count staining with nuclear fast red (Vector Laboratory, Cat\# H-3403).

\section{Acknowledgements}

We thank Dr. Yujiro Higashi's kindness for the Zeb1 mutant line. This work was supported by research grants from National Institutes of Health, USA (COBRE program of the National Center for Research Resource P20RR017702 to the University of Louisville Birth Defects Center, and DE016845 to JD) and by Kentucky Science and Engineering Foundation (KSEF-2070-RDE-013 to JD).

\section{References}

BLAVIER, L., LAZARYEV, A., GROFFEN, J., HEISTERKAMP, N., DECLERCK, Y.A. and KAARTINEN, V. (2001). TGF-beta3-induced palatogenesis requires matrix metalloproteinases. Mol Biol Cell 12: 1457-1466.

BUSH, J.O. and JIANG, R. (2012). Palatogenesis: morphogenetic and molecular mechanisms of secondary palate development. Development 139: 231-243.

CARETTE, M.J. and FERGUSON, M.W. (1992). The fate of medial edge epithelial cells during palatal fusion in vitro: an analysis by Dil labelling and confocal microscopy. Development 114: 379-388.

CHAI, Y. and MAXSON, R.E., JR. (2006). Recent advances in craniofacial morphogenesis. Dev Dyn 235: 2353-2375.

CUI, X.M., SHIOMI, N., CHEN, J., SAITO, T., YAMAMOTO, T., ITO, Y., BRINGAS, P., CHAI, Y. and SHULER, C.F. (2005). Overexpression of Smad2 in Tgf-beta3-null mutant mice rescues cleft palate. Dev Biol 278: 193-202.

ECONOMOU, A.D., OHAZAMA, A., PORNTAVEETUS, T., SHARPE, P.T., KONDO, S., BASSON, M.A., GRITLI-LINDE, A., COBOURNE, M.T. and GREEN, J.B. (2012). Periodic stripe formation by a Turing mechanism operating at growth zones in the mammalian palate. Nat Genet 44: 348-351.

FERGUSON, M.W. (1988). Palate development. Development 103 Suppl: 41-60.

FERGUSON, M.W., HONIG, L.S. and SLAVKIN, H.C. (1984). Differentiation of cultured palatal shelves from alligator, chick, and mouse embryos. Anat Rec 209: 231-249.

FITZPATRICK, D.R., DENHEZ, F., KONDAIAH, P. and AKHURST, R.J. (1990). Differential expression of TGF beta isoforms in murine palatogenesis. Development 109: 585-595.

GRIFFITH, C.M. and HAY, E.D. (1992). Epithelial-mesenchymal transformation during palatal fusion: carboxyfluorescein traces cells at light and electron microscopic levels. Development 116: 1087-1099.

HE, F., XIONG, W., YU, X., ESPINOZA-LEWIS, R., LIU, C., GU, S., NISHITA, M. SUZUKI, K., YAMADA, G., MINAMI, Y. et al., (2008). Wnt5a regulates directional cell migration and cell proliferation via Ror2-mediated noncanonical pathway in mammalian palate development. Development 135: 3871-3879.

HILLIARD, S.A., YU, L., GU, S., ZHANG, Z. and CHEN, Y.P. (2005). Regional regulation of palatal growth and patterning along the anterior-posterior axis in mice. $J$ Anat 207: 655-667.

JIN, J.Z. and DING, J. (2006). Analysis of Meox-2 mutant mice reveals a novel postfusion-based cleft palate. Dev Dyn 235: 539-546.

JIN, J.Z., LI, Q., HIGASHI, Y., DARLING, D.S. and DING, J. (2008). Analysis of Zfhx1a mutant mice reveals palatal shelf contact-independent medial edge epithelial differentiation during palate fusion. Cell Tissue Res 333: 29-38.

JIN, J.Z., TAN, M., WARNER, D.R., DARLING, D.S., HIGASHI, Y., GRIDLEY, T. and DING, J. (2010). Mesenchymal cell remodeling during mouse secondary palate reorientation. Dev Dyn 239: 2110-2117.

KAARTINEN, V., VONCKEN, J.W., SHULER, C., WARBURTON, D., BU, D., HEISTERKAMP, N. and GROFFEN, J. (1995). Abnormal lung development and cleft palate in mice lacking TGF-beta 3 indicates defects of epithelial-mesenchymal interaction. Nat Genet 11: 415-421. 
LI, Q. and DING, J. (2007). Gene expression analysis reveals that formation of the mouse anterior secondary palate involves recruitment of cells from the posterior side. Int J Dev Biol 51: 167-172.

MURRAY, J.C. and SCHUTTE, B.C. (2004). Cleft palate: players, pathways, and pursuits. J Clin Invest 113: 1676-1678.

NAWSHAD, A., LAGAMBA, D. and HAY, E.D. (2004). Transforming growth factor beta (TGFbeta) signalling in palatal growth, apoptosis and epithelial mesenchymal transformation (EMT). Arch Oral Biol 49: 675-689.

PELTON, R.W., HOGAN, B.L., MILLER, D.A. and MOSES, H.L. (1990). Differential expression of genes encoding TGFs beta 1, beta 2, and beta 3 during murine palate formation. Dev Biol 141: 456-460.

PROETZEL, G., PAWLOWSKI, S.A., WILES, M.V., YIN, M., BOIVIN, G.P., HOWLES, P.N., DING, J., FERGUSON, M.W. and DOETSCHMAN, T. (1995). Transforming growth factor-beta 3 is required for secondary palate fusion. Nat Genet 11:409-414.

SATOKATA, I. and MAAS, R. (1994). Msx1 deficient mice exhibit cleft palate and abnormalities of craniofacial and tooth development. Nat Genet 6: 348-356.

SHEN, M.M. (2001). Identification of differentially expressed genes in mouse devel- opment using differential display and in situ hybridization. Methods 24: 15-27.

SHULER, C.F., HALPERN, D.E., GUO, Y. and SANK, A.C. (1992). Medial edge epithelium fate traced by cell lineage analysis during epithelial-mesenchymal transformation in vivo. Dev Biol 154: 318-330.

TAKAGI, T., MORIBE, H., KONDOH, H. and HIGASHI, Y. (1998). DeltaEF1, a zinc finger and homeodomain transcription factor, is required for skeleton patterning in multiple lineages. Development 125: 21-31.

WELSH, I.C. and O'BRIEN, T.P. (2009). Signaling integration in the rugae growth zone directs sequential $\mathrm{SHH}$ signaling center formation during the rostral outgrowth of the palate. Dev Biol 336: 53-67.

YU, L., GU, S., ALAPPAT, S., SONG, Y., YAN, M., ZHANG, X., ZHANG, G., JIANG, Y., ZHANG, Z., ZHANG, Y. et al., (2005). Shox2-deficient mice exhibit a rare type of incomplete clefting of the secondary palate. Development 132: 4397-4406.

ZHANG, Z., SONG, Y., ZHAO, X., ZHANG, X., FERMIN, C. and CHEN, Y. (2002). Rescue of cleft palate in Msx1-deficient mice by transgenic Bmp4 reveals a network of BMP and Shh signaling in the regulation of mammalian palatogenesis. Development 129: 4135-4146. 


\section{Further Related Reading, published previously in the Int. J. Dev. Biol.}

Palatal adhesion is dependent on Src family kinases and p38MAPK

Yukiko Kitase and Charles F. Shuler

Int. J. Dev. Biol. (2014) 52: 289-289

http://dx.doi.org/10.1387/ijdb.130289yk

Microtubule disassembly prevents palatal fusion and alters regulation of the E-cadherin/catenin complex

Yukiko Kitase and Charles F. Shuler

Int. J. Dev. Biol. (2013) 57: 55-60

http://dx.doi.org/10.1387/ijdb.120117yk

TGF-beta3 is required for the adhesion and intercalation of medial edge epithelial cells during palate fusion

Consuelo Tudela, Miguel-Angel Formoso, Tamara Martínez, Raquel Pérez, Marta Aparicio, Carmen Maestro, Aurora Del Río, Elena Martínez, Mark Ferguson and Concepción Martínez-Alvarez

Int. J. Dev. Biol. (2002) 46: 333-336

http://dx.doi.org/10.1387/ijdb.12068957

The TGF-beta type III receptor is localized to the medial edge epithelium during palatal fusion $X \mathrm{M}$ Cui and C F Shuler

Int. J. Dev. Biol. (2000) 44: 397-402

http://dx.doi.org/10.1387/ijdb.10949049

Bulging medial edge epithelial cells and palatal fusion

C Martínez-Alvarez, R Bonelli, C Tudela, A Gato, J Mena, S O'Kane and M W Ferguson Int. J. Dev. Biol. (2000) 44: 331-335

http://dx.doi.org/10.1387/ijdb.10853831

Immunohistochemical localization of TGF-beta type II receptor and TGF-beta3 during palatogenesis in vivo and in vitro

X M Cui, D Warburton, J Zhao, D L Crowe and C F Shuler

Int. J. Dev. Biol. (1998) 42: 817-820

http://dx.doi.org/10.1387/ijdb.9727838

Inhibition of TGF-beta 3 (but not TGF-beta 1 or TGF-beta 2) activity prevents normal mouse embryonic palate fusion

C L Brunet, P M Sharpe and M W Ferguson

Int. J. Dev. Biol. (1995) 39: 345-355

http://dx.doi.org/10.1387/ijdb.7669547

5 yr ISI Impact Factor $(2013)=2.879$
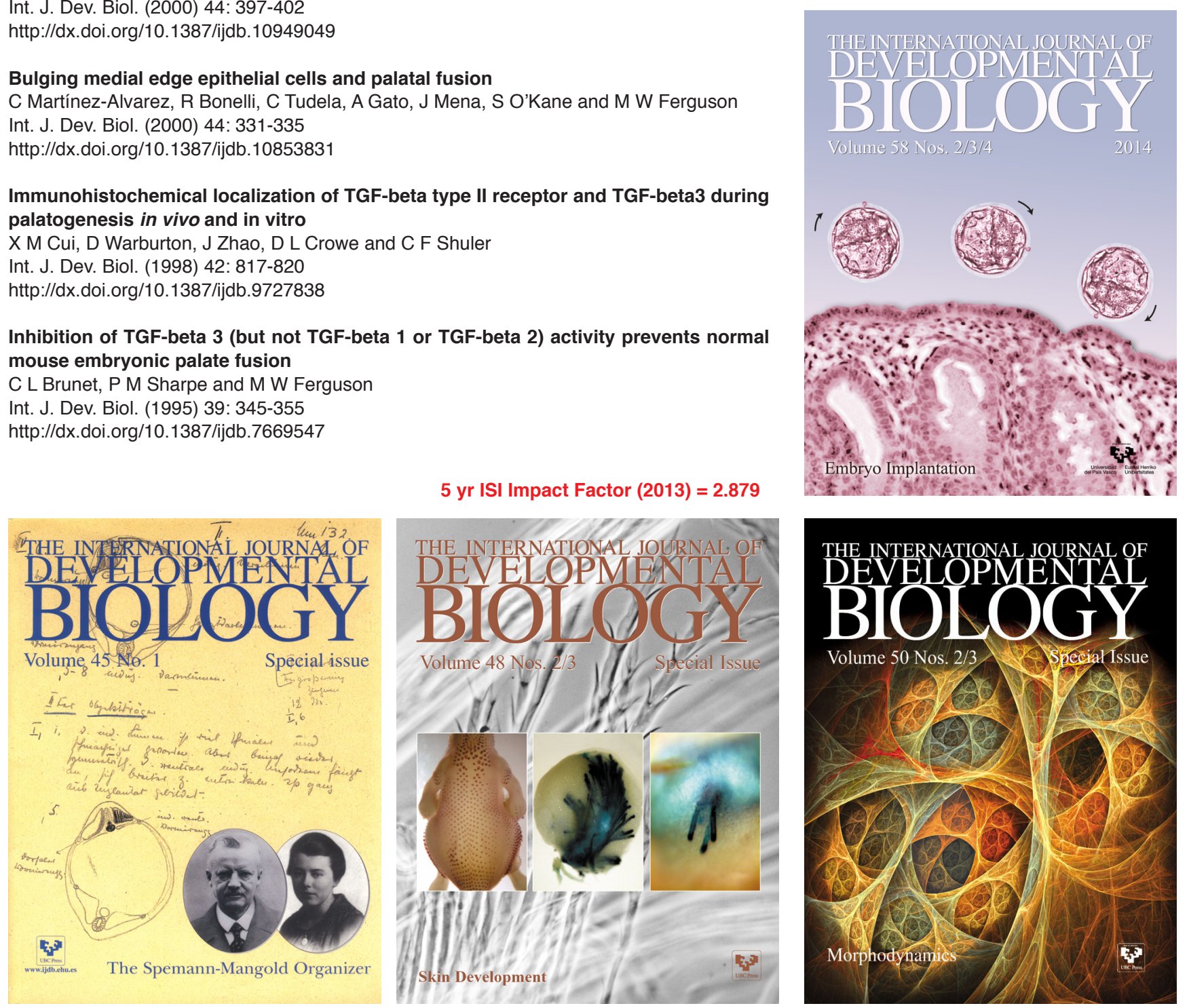